COMPUTATIONAL METHODS IN APPLIED MATHEMATICS, Vol.1(2001), No.4, pp.319-332

(C) Institute of Mathematics of the National Academy of Sciences of Belarus

\title{
NUMERICAL APPROXIMATION OF THE WEAKLY DAMPED NONLINEAR SCHRÖDINGER EQUATION
}

\author{
RAIMONDAS ČIEGIS \\ Vilnius Gediminas Technical University \\ Saulètekio al. 11, LT-2040 Vilnius, Lithuania \\ E-mail: rc@fm.vtu.lt \\ VIOLETA PAKENIENE் \\ Institute of Mathematics and Informatics \\ Akademijos 4, LT-2600 Vilnius, Lithuania \\ E-mail: vp@fm.vtu.It
}

\begin{abstract}
In this paper we consider the one-dimensional nonlinear Schrödinger equation. The equation includes an absorption term, and the solution is periodically amplified in order to compensate the lose of the energy. The problem describes propagation of a signal in optical fibers. In our previous work we proved that the well-known Crank-Nicolson scheme is unconditionally unstable for this problem. We present in this paper two finite difference approximations. The first one is given by a modified Crank-Nicolson scheme and the second one is obtained by a splitting scheme. The stability and convergence of these schemes are proved. The results of numerical experiments are presented and discussed.
\end{abstract}

2000 Mathematics Subject Classification: 65N20; 65M25.

Keywords: finite difference scheme, nonlinear Schrödinger equation, splitting method, stability.

\section{Introduction}

We consider the weakly damped nonlinear Schrödinger equation [12]

$$
i \frac{\partial u}{\partial z}+\frac{1}{2} \frac{\partial^{2} u}{\partial t^{2}}+\mu|u|^{2} u+i \alpha u=0 \quad \text { in }(0, L] \times(0, T] .
$$

Equation (1) is supplemented with the boundary conditions

$$
u(z, 0)=0, \quad u(z, T)=0 \quad \text { on }[0, L]
$$

and initial conditions

$$
u(0, t)=u_{0}(t) \quad \text { in }[0, T] .
$$


It describes the propagation of a binary signal by solitons through optical fibers. Here $z$ is the abscissa along the fiber, $t$ the time, $\alpha$ the absorption coefficient, a real positive parameter, and $u$ is a complex-valued function of $t$ and $z$.

The signal is periodically amplified along the fiber at the distances which are multiple of $z_{a}$ :

$$
u\left(k z_{a}, t\right)=e^{\alpha z_{a}} u\left(k z_{a}-0, t\right) .
$$

Each amplification operation implies the addition of a white noise interference, but we do not consider this stochastic process (see [12]).

The initial condition describes a sequence of soliton-type pulses:

$$
u_{0}(t)=\sum_{j=0}^{P} \frac{a_{j}}{\operatorname{ch}(t-8 j-4)},
$$

where $\left\{a_{j}\right\}$ is a sequence defining the binary information, i.e., $a_{j}=1$ or $a_{j}=0$.

Finite difference and finite element schemes for solving the linear and nonlinear Schrödinger equation have been extensively studied in many papers, see, e.g., [1, 5, 6, 8, 14, 17]. The weakly damped nonlinear Schrödinger equation was investigated in particular in $[9,12]$. The problem is solved by splitting-type schemes, and no convergence results are given in these papers.

We are interested in the stability and convergence analysis of finite difference schemes for solving the nonlinear Schrödinger equation, which additionally includes a combination of absorption and amplification processes. It is well known that the stability results obtained for discrete approximations of separate physical phenomena can be insufficient when a combination of such processes is considered. See [18] for the analysis of nonstationary diffusion-advection equations.

We proved in $[3,4]$ that even in a linear case a careful balance of absorption and amplification processes must be preserved in order to get unconditionally stable finite difference schemes. In this paper we extend these results to nonlinear problem (1) - (4).

The outline of this paper is as follows. In Section 2, we propose and investigate a modified Crank-Nicolson approximation. In Section 3, we present a splitting-type approximation of the problem and investigate the stability of this scheme. Finally, in Section 4, we present the results of some numerical experiments.

\section{Finite difference scheme}

Let $\omega_{h}$ be a family of uniform spatial discrete grids

$$
\omega_{h}=\left\{z^{n}: z^{n}=n h, \quad n=1,2, \ldots, M, z^{M}=L\right\},
$$

and let $\omega_{\tau}$ be a family of uniform temporal grids

$$
\omega_{\tau}=\left\{t_{j}: t_{j}=j \tau, \quad j=1,2, \ldots, N-1\right\}, \quad t_{N}=T .
$$

We denote by $y_{j}^{n}=y\left(z^{n}, t_{j}\right)$ a discrete function defined on $\omega_{h} \times \omega_{\tau}$. The following common notation of difference derivatives is used in our paper (see [13]):

$$
y_{z}=\frac{y^{n+1}-y^{n}}{h}, \quad y_{\bar{t}}=\frac{y_{j}-y_{j-1}}{\tau}, \quad y_{t}=\frac{y_{j+1}-y_{j}}{\tau} .
$$


We define two time inner products and three norms of discrete functions:

$$
\begin{aligned}
& (y, v)=\sum_{j=1}^{N-1} y_{j} v_{j}^{*} \tau, \quad(y, v]=\sum_{j=1}^{N} y_{j} v_{j}^{*} \tau \\
& \left.\|y\|^{2}=(y, y), \quad \| y_{\bar{t}}\right]\left.\right|^{2}=\sum_{j=1}^{N} y_{j, \bar{t}}^{2} \tau \\
& \|y\|_{C}=\max _{1 \leqslant j<N}\left|y_{j}\right| .
\end{aligned}
$$

Many popular approximations of the nonlinear Schrödinger equation are obtained using the Crank-Nicolson method. In the case of nonlinear damped equation (1) we get the following scheme:

$$
\begin{gathered}
i \frac{y^{n+1}-y^{n}}{h}+\frac{1}{2}\left(\frac{y^{n+1}+y^{n}}{2}\right)_{\bar{t} t}+\mu \frac{\left|y^{n+1}\right|^{2}+\left|y^{n}\right|^{2}}{2} \frac{y^{n+1}+y^{n}}{2}+i \alpha \frac{y^{n+1}+y^{n}}{2}=0, t_{j} \in \omega_{\tau}, \\
y_{0}^{n}=0, \quad y_{N}^{n}=0, \quad z^{n} \in \omega_{h}, \\
y\left(z^{0}, t_{j}\right)=u_{0}\left(t_{j}\right), \quad t_{j} \in \omega_{\tau} .
\end{gathered}
$$

The amplification condition (4) is approximated as follows:

$$
y_{j}^{n}=\left(\frac{1+0.5 \alpha h}{1-0.5 \alpha h}\right)^{K} y\left(z^{n}-0, t_{j}\right),
$$

where $z^{n}=m z_{a}$ coincides with an amplification point and $K=z_{a} / h$.

In our previous work [3] we have proved that scheme (5) - (6) is unconditionally unstable for the linear case of problem (1) - (4), i.e., when $\mu=0$. In this paper we propose two schemes for solving the problem. The first scheme is a modification of the Crank-Nicolson scheme, and the second one is obtained by using the splitting method.

\subsection{Modified finite difference scheme}

In this section we consider a modification of scheme (5). Let $v$ be a new unknown function, which is defined by the equality

$$
u(z, t)=e^{-\alpha\left(z-z_{m}\right)} v(z, t), \quad z_{m} \leqslant z \leqslant z_{m+1},
$$

here $z_{m}=m z_{a}$ are the amplification points. Substituting this equality into equation (1), we get the nonlinear Schrödinger equation for the function $v$ :

$$
i \frac{\partial v}{\partial z}+\frac{1}{2} \frac{\partial^{2} v}{\partial t^{2}}+\mu e^{-2 \alpha\left(z-z_{m}\right)}|v|^{2} v=0 \quad \text { in }(0, L] \times(0, T]
$$

which is supplemented with appropriate boundary conditions

$$
v(z, 0)=0, \quad v(z, T)=0 \quad \text { on }[0, L],
$$

and initial conditions

$$
v\left(z_{m}, t\right)=u\left(z_{m}, t\right) \quad \text { on }[0, T] .
$$


It follows from the amplification condition (4) that

$$
\begin{aligned}
u\left(z_{m+1}, t\right) & =e^{\alpha z_{a}} u\left(z_{m+1}-0, t\right) \\
& =e^{\alpha z_{a}} e^{-\alpha\left(z_{m+1}-z_{m}\right)} v\left(z_{m+1}, t\right)=v\left(z_{m+1}, t\right) .
\end{aligned}
$$

Hence, condition (9) is satisfied at $z=z_{m+1}$ after the amplification step, and the whole procedure can be repeated in the interval $\left[z_{m+1}, z_{m+2}\right]$.

The finite difference approximation of $(7)-(9)$ is defined as follows:

$$
\begin{gathered}
i \frac{w^{n+1}-w^{n}}{h}+\frac{1}{2}\left(\frac{w^{n+1}+w^{n}}{2}\right)_{\bar{t} t}+\mu e^{-2 \alpha\left(z^{n+1 / 2}-z_{m}\right)} \frac{\left|w^{n+1}\right|^{2}+\left|w^{n}\right|^{2}}{2} \frac{w^{n+1}+w^{n}}{2}=0, t_{j} \in \omega_{\tau}, \\
w_{0}^{n}=0, \quad w_{N}^{n}=0, \quad z^{n} \in \omega_{h}, \\
w\left(z^{0}, t_{j}\right)=u_{0}\left(t_{j}\right), \quad t_{j} \in \omega_{\tau} .
\end{gathered}
$$

We use the discrete function

$$
y_{j}^{n}= \begin{cases}e^{-\alpha\left(z^{n}-z_{m}\right)} w_{j}^{n} & \text { for } z_{m} \leqslant z^{n}<z_{m+1}, \\ w_{j}^{n} & \text { for } z^{n}=z_{m+1}\end{cases}
$$

as the approximation of the solution of problem (1) - (4). In order to find a solution of (10) we use the following conservative iterative algorithm:

$$
\begin{aligned}
& i \frac{\stackrel{\mathrm{s}}{w}-w^{n}}{h}+\frac{1}{2}\left(\frac{\stackrel{\mathrm{s}}{w}+w^{n}}{2}\right)_{\bar{t} t}+\mu e^{-2 \alpha\left(z^{n+1 / 2}-z_{m}\right)} \frac{|\stackrel{\mathrm{s}-1}{w}|^{2}+\left|w^{n}\right|^{2}}{2} \frac{\stackrel{\mathrm{s}}{w}+w^{n}}{2}=0, \\
& \stackrel{0}{w}=w^{n},
\end{aligned}
$$

here $\stackrel{\mathrm{s}}{w}$ defines a sequence of iterations. Taking the inner product of (11) with $\stackrel{\mathrm{s}}{w}+w^{n}$ we obtain that the full energy of the solution is preserved in each iteration

$$
\|\stackrel{\mathrm{s}}{w}\|^{2}=\left\|w^{n}\right\|^{2}, \quad s=1,2, \cdots, S .
$$

\subsection{Convergence analysis}

The convergence analysis of finite difference scheme (10) is based on the methodology firstly proposed in [2]. Let us consider two auxiliary boundary-value problems for the functions $\mathrm{Y}$ and $\mathrm{V}$ :

$$
\begin{aligned}
& i \frac{Y-y}{h}+ \frac{1}{2}\left(\frac{Y+y}{2}\right)_{\bar{t} t}+\mu e^{-2 \alpha\left(z^{n+1 / 2}-z_{m}\right)} \\
& \times\left(\gamma \frac{|g|^{2}+|y|^{2}}{2}+(1-\gamma) \frac{|Y|^{2}+|y|^{2}}{2}\right) \frac{Y+y}{2}=G, \quad t_{j} \in \omega_{\tau}, \\
& Y_{0}=0, \quad Y_{N}=0 ; \\
& i \frac{V-v}{h}+ \frac{1}{2}\left(\frac{V+v}{2}\right)_{\bar{t} t}+\mu e^{-2 \alpha\left(z^{n+1 / 2}-z_{m}\right)} \\
& \times\left(\gamma \frac{|p|^{2}+|v|^{2}}{2}+(1-\gamma) \frac{|V|^{2}+|v|^{2}}{2}\right) \frac{V+v}{2}=0, \quad t_{j} \in \omega_{\tau}, \\
& V_{0}=0, \quad V_{N}=0,
\end{aligned}
$$


here $y, v, g, G$, and $p$ are given discrete functions.

Let us assume that the following two sets of a priori conditions are satisfied:

$$
\begin{aligned}
& \left.\left.|| y_{\bar{t}}\right] \mid \leqslant C_{1}, \quad \| v_{\bar{t}}\right]\left|\leqslant C_{1}, \quad \| g_{\bar{t}}\right|\left|\leqslant C_{2}, \quad\right|\left|p_{\bar{t}}\right| \mid \leqslant C_{2} \\
& y_{0}=0, \quad y_{N}=0, \quad v_{0}=0, \quad v_{N}=0, \\
& g_{0}=0, \quad g_{N}=0, \quad p_{0}=0, \quad p_{N}=0, \\
& \left.\| Y_{\bar{t}}\right]\left|\leqslant C_{2}, \quad \| V_{\bar{t}}\right| \leqslant C_{2} .
\end{aligned}
$$

First, we will investigate the stability of nonlinear finite difference scheme (10).

Lemma 2.1. Let us assume that the functions $Y$ and $V$ are the solutions of problems (12), (13), respectively. If $\gamma=1$ and a priori conditions $(A)$ are satisfied, then the following estimate

$$
\left.\left.\left.\left.\| Y_{\bar{t}}-V_{\bar{t}}\right]\left.\right|^{2} \leqslant\left(1+C_{3} h\right) \| y_{\bar{t}}-v_{\bar{t}}\right]\left.\right|^{2}+C_{4} h \| g_{\bar{t}}-z_{\bar{t}}\right]\left.\right|^{2}+C_{5} h \| G_{\bar{t}}\right] \mid
$$

is valid; here $C_{3}, C_{4}$, and $C_{5}$ are constants which may depend on $C_{1}$ and $C_{2}$ but they do not depend on the discrete steps $h$ and $\tau$.

Proof. The proof is similar to that given in [1]. We subtract equation (13) from equation (12), take the inner product of the obtained equality with $2(Y-V)_{\bar{t} t}$, and apply the summation-by-parts formula to all the terms, excluding only the dispersion term. Then we consider the imaginary part of the equation. Applying the embedding theorem

$$
\|y\|_{C} \leqslant \frac{1}{2}|| y_{\bar{t}} \mid
$$

to the nonlinear terms, using the Schwarz inequality, and applying assumptions $(A)$, we prove the lemma.

Lemma 2.2. Let us assume that the assumptions of Lemma 2.1 are satisfied and conditions $(B)$ are valid. Then, for $\gamma=0$ and $h$ sufficiently small the following estimate

$$
\left.\left.\| Y_{\bar{t}}-\left.V_{\bar{t}}\right|^{2} \leqslant\left(1+C_{6} h\right)|| y_{\bar{t}}-v_{\bar{t}}\right]\left.\right|^{2}+C_{7} h|| G_{\bar{t}}\right] \mid
$$

holds; here the constants $C_{6}$ and $C_{7}$ do not depend on the discrete steps $h$ and $\tau$.

Proof. Taking in Lemma $2.1 \mathrm{~g}=Y, p=V$ and using conditions $(B)$, we get from (14) that

$$
\left.\left.\left(1-C_{4} h\right)|| Y_{\bar{t}}-\left.V_{\bar{t}}\right|^{2} \leqslant\left(1+C_{3} h\right)|| y_{\bar{t}}-v_{\bar{t}}\right]\left.\right|^{2}+C_{5} h|| G_{\bar{t}}\right] \mid .
$$

Taking $h$ sufficiently small, i.e., such that $h \leqslant h_{0}=0.5 C_{4}$, after simple computations we prove the stability inequality (15) with the following constants $C_{6}=2\left(C_{3}+C_{4}\right), C_{7}=$ $2 C_{5}$.

By using Lemma 2.1 and Lemma 2.2, we can investigate the convergence of the modified difference scheme (10).

The correctness of the finite difference scheme is proved by induction. Let us assume that $w^{n}$ exists and it satisfies the a priori conditions $(A)$. 
Lemma 2.3. For $h$ sufficiently small, all iterations $\stackrel{\mathrm{s}}{w}$ from $(11)$ are uniformly bounded

$$
\left.\| \stackrel{\mathrm{s}}{w_{\bar{t}}}\right] \mid \leqslant C_{2}=2 C_{1} .
$$

Proof. Taking in Lemma 2.1

$$
Y=\stackrel{\mathrm{s}}{w}, \quad y=w^{n}, \quad g=\stackrel{\mathrm{s}-1}{w}, \quad V=v=p=0, G=0,
$$

we get from (14) that

$$
\left.\| \stackrel{\mathrm{s}}{w}_{\bar{t}}^{\mathrm{s}}\right]\left.\right|^{2} \leqslant\left(1+C_{3} h\right) C_{1}^{2}+4 C_{4} C_{1}^{2} h
$$

Then, the required estimate follows immediately for sufficiently small spatial grid steps:

$$
h \leqslant \frac{1}{C}, \quad C=\max \left(C_{3}, 2 C_{4}\right) .
$$

Lemma 2.4. For h sufficiently small the iteration sequence $\stackrel{\mathrm{s}}{w}$ defines a Cauchy sequence.

Proof. Taking in Lemma 2.1

$$
Y=\stackrel{\mathrm{s}+1}{w}, \quad y=v, \quad g=V=\stackrel{\mathrm{s}}{w}, \quad p=\stackrel{\mathrm{s}-1}{w}, \quad G=0
$$

and assuming that $h \leqslant 1 /\left(2 C_{4}\right)$, we obtain from (14) that

$$
\left.\left.\left.\|(\stackrel{\mathrm{s}+1}{w}-\stackrel{\mathrm{s}}{w})_{\bar{t}}\right]\left.\right|^{2} \leqslant C_{4} h \|(\stackrel{\mathrm{s}}{w}-\stackrel{\mathrm{s}-1}{w}) \bar{t}\right]\left.\right|^{2} \leqslant \frac{1}{2}||(\stackrel{\mathrm{s}}{w}-\stackrel{\mathrm{s}-1}{w}) \bar{t}\right]\left.\right|^{2} .
$$

The lemma is proved.

Using the properties of Cauchy sequences, we get that $\stackrel{s}{w}$ converges to the limit

$$
\lim _{s \rightarrow \infty} \stackrel{\mathrm{s}}{w}=w^{n+1}
$$

and this limit function is bounded by the same constant $C_{2}$ :

$$
\left.|| w_{\bar{t}}^{n+1}\right] \mid \leqslant C_{2} .
$$

Lemma 2.5. For h sufficiently small, finite difference scheme (10) has a unique solution.

Proof. Let us assume that there exist two solutions $w^{n+1}$ and $v^{n+1}$ of (10). Then taking in Lemma 2.2

$$
Y=w^{n+1}, y=w^{n}, V=v^{n+1}, v=w^{n}, G=0,
$$

we obtain from (15) that

$$
\left.\|\left(w^{n+1}-v^{n+1}\right) \bar{t}\right]\left.\right|^{2} \leqslant 0 .
$$

This inequality can be satisfied only if $w^{n+1}=v^{n+1}$. The lemma is proved.

Lemma 2.6. For $h$ sufficiently small the following stability estimate

$$
\left.\left.\|\left(w^{n}-v^{n}\right)_{\bar{t}}\right] \mid \leqslant \sqrt{C_{7}} e^{0.5 C_{6} z^{n}} \max _{1 \leqslant j \leqslant n} \| \Psi_{\bar{t}}^{j}\right] \mid
$$

is satisfied, where $\Psi^{j}$ is a truncation error. 
Proof. Taking in Lemma 2.2

$$
Y=v^{n+1}, y=v^{n}, V=w^{n+1}, v=w^{n}, G=\Psi^{n+1},
$$

we get from the (15) that

$$
\left.\left.\|\left(w^{n+1}-v^{n+1}\right)_{\bar{t}}\right]\left.\right|^{2} \leqslant\left(1+C_{6} h\right)\left\|\left.\left(w^{n}-v^{n}\right)_{\bar{t}}\right|^{2}+C_{7} h\right\| \Psi_{\bar{t}}^{n+1}\right]\left.\right|^{2} .
$$

Then, the proof of the required stability estimate follows in view of the Gronwall discrete inequality.

Lemma 2.7. Let us assume that $v \in C_{4}^{4}$, then the truncation error of finite difference scheme (10) satisfies the following estimate:

$$
\left.|| \Psi_{\bar{t}}^{n+1}\right] \mid \leqslant C\left(h^{2}+\tau^{2}\right) .
$$

Proof. We extend the definition of the solution $v$ and the discrete solution $w_{j}$ outside of the grid $\omega_{\tau}$ by using the odd function property

$$
\begin{array}{r}
v(z,-t)=-v(z, t), \quad v(z, T+t)=-v(z, T-t), \\
w_{-1}=-w_{1}, \quad w_{N+1}=-w_{N-1} .
\end{array}
$$

Then, finite difference scheme (10) is satisfied also on the boundary of $\omega_{\tau}$. Expanding the solution $v(z, t)$ in a Taylor series expansion and using the Lagrange form of the remainder term, we get the required estimate.

The main result of this section is given in the following theorem.

Theorem 2.1. For $h$ sufficiently small, finite difference scheme (10) has a unique solution, which can be defined by iterative process (11), and the global error of the discrete solution satisfies the estimate

$$
\left\|\left(w^{n}-u^{n}\right) \bar{t}\right\| \leqslant C\left(\tau^{2}+h^{2}\right) .
$$

\section{Splitting scheme}

Let $\omega_{h}^{m}$ be a subgrid of the main space grid $\omega_{h}$ :

$$
w_{h}^{m}=\left\{Z^{n}: \quad Z^{n}=z_{m-1}+n h, \quad n=1,2, \ldots, K, \quad Z^{K}=z_{m}\right\}
$$

here $z_{m}=m z_{\alpha}$ are the amplification points. We introduce a new discrete function $V^{m}$, which is defined at the amplification points $z_{m}$.

In order to construct the finite difference scheme, we split the problem (1)-(4) into three parts: the first part includes the absorption process, the second describes the diffraction and nonlinear interaction processes, and the third takes into account the amplification of the solution. Each part is solved numerically separately and efficient schemes can be applied. For the mathematical theory of splitting schemes as well as for related computational algorithms we refer the readers to $[11,13,18]$. 
The solution $y^{n+1}$ on $w_{h}^{m}$ is computed by using the implicit scheme

$$
\begin{gathered}
\frac{y^{n+1 / 2}-y^{n}}{h}+\alpha \frac{y^{n+1 / 2}+y^{n}}{2}=0 \\
i \frac{y^{n+1}-y^{n+1 / 2}}{h}+\frac{1}{2}\left(\frac{y^{n+1}+y^{n+1 / 2}}{2}\right)_{\bar{t} t}+\mu \frac{\left|y^{n+1}\right|^{2}+\left|y^{n+1 / 2}\right|^{2}}{2} \frac{y^{n+1}+y^{n+1 / 2}}{2}=0
\end{gathered}
$$

which is supplemented with appropriate boundary and initial conditions

$$
\begin{gathered}
y_{0}^{n}=0, \quad y_{N}^{n}=0, \quad Z^{n} \in \omega_{h}^{m}, \\
y\left(Z^{0}, t_{j}\right)=V_{j}^{m-1},
\end{gathered}
$$

The amplification condition is approximated by the discrete equation

$$
V_{j}^{m}=\left(\frac{1+0.5 \alpha h}{1-0.5 \alpha h}\right)^{K} y_{j}^{K} .
$$

It follows from (16) that

$$
\left\|y^{n+1 / 2}\right\|=\left|\frac{1-0.5 \alpha h}{1+0.5 \alpha h}\right|\left\|y^{n}\right\|
$$

Taking the inner product of (17) with $y^{n+1 / 2}+y^{n+1}$ and investigating the imaginary part, we obtain $\left\|y^{n+1}\right\|=\left\|y^{n+1 / 2}\right\|$. Combining both equalities, we have

$$
\left\|y^{n+1}\right\|=\left|\frac{1-0.5 \alpha h}{1+0.5 \alpha h}\right|\left\|y^{n}\right\| \text {. }
$$

Iterating the obtained equality, we see that

$$
\left\|y^{K}\right\|=\left|\frac{1-0.5 \alpha h}{1+0.5 \alpha h}\right|^{K}\left\|V^{m-1}\right\| .
$$

Then, using (18), we obtain the required equality $\left\|V^{m}\right\|=\left\|V^{m-1}\right\|$. Thus, the scheme is conservative.

Equation (16) can be easily solved in an explicit form

$$
y^{n+1 / 2}=\frac{1-0.5 \alpha h}{1+0.5 \alpha h} y^{n} .
$$

Nonlinear problem (17) is solved by using the iterative algorithm

$$
\frac{\stackrel{\mathrm{s}}{y-y^{n+1 / 2}}}{h}+\frac{1}{2}\left(\frac{\stackrel{\mathrm{s}}{y}+y^{n+1 / 2}}{2}\right)_{\bar{t} t}+\mu \frac{\left.\left.\right|^{\mathrm{s}-1}\right|^{2}+\left|y^{n+1 / 2}\right|^{2}}{2} \frac{\stackrel{\mathrm{s}}{y}+y^{n+1 / 2}}{2}=0 .
$$

Taking the inner product of (20) with $\stackrel{\mathrm{s}}{y}+y^{n+1 / 2}$, we obtain that $\|\stackrel{\mathrm{s}}{y}\|=\left\|y^{n+1 / 2}\right\|$, i.e., the iterative algorithm is conservative.

The convergence of the discrete solution is proved by induction. Let us assume that the following inequalities

$$
\left.\left.|| V_{\bar{t}}^{m-1}\right] \mid \leqslant C_{1}, \quad \| y_{\bar{t}}^{n}\right] \mid \leqslant C_{1}
$$


are valid. Then, the solution representation (19) immediately leads us to an estimate

$$
\left.|| y_{\bar{t}}^{n+1 / 2}\right] \mid \leqslant C_{1}
$$

The existence and uniqueness of the solution of (17) follows from Lemma 2.1 and Lemma 2.2.

Now, we will prove the main stability inequality. The proof is divided into two parts. First, we will get the recurrence relation for the error functions

$$
e^{n}=u^{n}-y^{n}, \quad e^{n+1 / 2}=u^{n+1}-y^{n+1 / 2} .
$$

Then, we will bound the global error function $E^{m}=u\left(z_{m}, t\right)-V^{m}$.

\subsection{Stability analysis for $e^{n}$}

The error function $e^{n}$ satisfies the following finite difference scheme:

$$
\begin{aligned}
\frac{e^{n+1 / 2}-e^{n}}{h} & +\alpha \frac{e^{n+1 / 2}+e^{n}}{2}=\psi_{1}^{n+1}, \\
i \frac{e^{n+1}-e^{n+1 / 2}}{h} & +\frac{1}{2}\left(\frac{e^{n+1}+e^{n+1 / 2}}{2}\right)_{\bar{t} t}+\mu\left(f_{1}\left(u^{n+1}, u^{n}, y^{n+1}, y^{n}\right) e^{n+1}\right. \\
& +f_{2}\left(u^{n+1}, u^{n}, y^{n+1}, y^{n}\right)\left(e^{n+1}\right)^{*}+f_{3}\left(u^{n+1}, u^{n}, y^{n+1}, y^{n}\right) e^{n} \\
& \left.+f_{4}\left(u^{n+1}, u^{n}, y^{n+1}, y^{n}\right)\left(e^{n}\right)^{*}\right)=\psi_{2}^{n+1},
\end{aligned}
$$

there $\psi_{k}^{n+1}$ are the truncation errors and $f_{k}$ are the quadratic polynomials:

$$
\begin{aligned}
& f_{1}(a, b, c, d)=\frac{1}{4}\left(a a^{*}+b b^{*}+c c^{*}+d c^{*}\right), \quad f_{2}(a, b, c, d)=\frac{1}{4}(a c+a d), \\
& f_{3}(a, b, c, d)=\frac{1}{4}\left(a a^{*}+b b^{*}+c d^{*}+d d^{*}\right), \quad f_{4}(a, b, c, d)=\frac{1}{4}(b c+b d) .
\end{aligned}
$$

We split the truncation errors into two parts (see [13])

$$
\psi_{k}^{n+1}=\Psi_{k}^{0}+\Psi_{k}^{1}, \quad k=1,2,
$$

where

$$
\begin{aligned}
& \Psi_{1}^{0}=\frac{\partial u^{n+1}}{\partial z}+\alpha u^{n+1}=\mathcal{O}(1) \\
& \Psi_{2}^{0}=\frac{1}{2} \frac{\partial^{2} u^{n+1}}{\partial t^{2}}+\mu\left|u^{n+1}\right|^{2} u^{n+1}=\mathcal{O}(1)
\end{aligned}
$$

and for $u \in C_{4}^{3}$

$$
\Psi_{1}^{1}=\mathcal{O}\left(h^{2}\right), \quad \Psi_{2}^{1}=\mathcal{O}\left(h^{2}+\tau^{2}\right) .
$$

Using $(23)-(24)$, we obtain that the functions $\Psi_{k}^{0}$ satisfy the equality

$$
\Psi_{1}^{0}+\Psi_{2}^{0}=0
$$

Lemma 3.1. The finite difference scheme (16) - (17) admits the a priori error estimate

$$
\left.\left.\left.\left.\| e_{\bar{t}}^{n+1}\right]\left.\right|^{2} \leqslant(1+C h)^{2} q^{2} \| e_{\bar{t}}^{n}\right]\left.\right|^{2}+C h \| \Phi_{\bar{t}}^{m, n+1 / 2}\right]\left.\right|^{2}+C h \| \Phi_{\bar{t}}^{m, n+1}\right]\left.\right|^{2},
$$


where

$$
q=\frac{1-0.5 \alpha h}{1+0.5 \alpha h}
$$

and $\Phi^{m, n+k / 2}$ depends on truncation errors:

$$
\begin{aligned}
\Phi^{m, n+1 / 2}= & \Psi_{1}^{1}-\frac{1}{2} \alpha h \Psi_{1}^{0}, \\
\Phi^{m, n+1}= & \Psi_{2}^{1}-\frac{1}{2} i h \Psi_{1, \bar{t} t}^{0}-\mu h\left(f_{3}\left(u^{n+1}, u^{n}, y^{n+1}, y^{n}\right) \Psi_{1}^{0}\right. \\
& \left.+f_{4}\left(u^{n+1}, u^{n}, y^{n+1}, y^{n}\right)\left(\Psi_{1}^{0}\right)^{*}\right) .
\end{aligned}
$$

Proof. We write the error function $e^{n}$ as a sum of two new functions (see [13])

$$
e^{n+k / 2}=\xi^{n+k / 2}+\eta^{n+k / 2}, \quad k=1,2 .
$$

The function $\xi^{n+k / 2}$ is a solution of the auxiliary finite difference scheme

$$
\begin{gathered}
\frac{\xi^{n+1 / 2}-\xi^{n}}{h}=\Psi_{1}^{0}, \\
\frac{\xi^{n+1}-\xi^{n+1 / 2}}{h}=\Psi_{2}^{0},
\end{gathered}
$$

supplemented with the initial condition

$$
\xi^{0}=0 .
$$

Due to equality (25) we obtain that

$$
\xi^{n+1}=\xi^{n}+h\left(\Psi_{1}^{0}+\Psi_{2}^{0}\right)=\xi^{n} .
$$

The initial condition yields that

$$
\xi^{n}=0, \quad n=1,2, \cdots, K .
$$

For $\xi^{n+1 / 2}$ we easily get the bound

$$
\xi^{n+1 / 2}=h \Psi_{1}^{0}=\mathcal{O}(h) .
$$

The functions $\eta^{n+k / 2}$ satisfy the following finite difference problem:

$$
\begin{gathered}
\frac{\eta^{n+1 / 2}-\eta^{n}}{h}+\alpha \frac{\eta^{n+1 / 2}+\eta^{n}}{2}=\Phi^{m, n+1 / 2} \\
\Phi^{m, n+1 / 2}=\Psi_{1}^{1}-\frac{1}{2} \alpha h \Psi_{1}^{0}, \\
i \frac{\eta^{n+1}-\eta^{n+1 / 2}}{h}+\frac{1}{2}\left(\frac{\eta^{n+1}+\eta^{n+1 / 2}}{2}\right)_{\bar{t} t}+\mu\left(f_{1}\left(u^{n+1}, u^{n}, y^{n+1}, y^{n}\right) \eta^{n+1}\right. \\
+f_{2}\left(u^{n+1}, u^{n}, y^{n+1}, y^{n}\right)\left(\eta^{n+1}\right)^{*}+f_{3}\left(u^{n+1}, u^{n}, y^{n+1}, y^{n}\right) \eta^{n} \\
\left.+f_{4}\left(u^{n+1}, u^{n}, y^{n+1}, y^{n}\right)\left(\eta^{n}\right)^{*}\right)=\Phi^{n+1}, \\
\Phi^{m, n+1}=\Psi_{2}^{1}-\frac{1}{2} i h \Psi_{1, \bar{t} t}^{0}-\mu h\left(f_{3}\left(u^{n+1}, u^{n}, y^{n+1}, y^{n}\right) \Psi_{1}^{0}\right. \\
\left.+f_{4}\left(u^{n+1}, u^{n}, y^{n+1}, y^{n}\right)\left(\Psi_{1}^{0}\right)^{*}\right) .
\end{gathered}
$$


It follows from (28) that

$$
\left.\left.\left.\| \eta_{\bar{t}}^{n+1 / 2}\right]|\leqslant| q|| \mid \eta_{\bar{t}}^{n}\right]\left|+\frac{h}{1+0.5 \alpha h}\right| \mid \Phi_{\bar{t}}^{m, n+1 / 2}\right] \mid .
$$

Using the Schwarz inequality, and for $h$ sufficiently small, after simple computations we get

$$
\left.\left.\left.\| \eta_{\bar{t}}^{n+1 / 2}\right]\left.\right|^{2} \leqslant q^{2}(1+C h)|| \eta_{\bar{t}}^{n}\right]\left.\right|^{2}+\frac{C_{3} h}{(1+0.5 \alpha h)^{2}} \| \Phi_{\bar{t}}^{m, n+1 / 2}\right]\left.\right|^{2} .
$$

Similarly to the proof of Lemma 2.6 we get from (29) the stability inequality

$$
\left.\left.\left.\| \eta_{\bar{t}}^{n+1}\right]\left.\right|^{2} \leqslant(1+C h)|| \eta_{\bar{t}}^{n+1 / 2}\right]\left.\right|^{2}+C h|| \Phi_{\bar{t}}^{m, n+1}\right]\left.\right|^{2} .
$$

Inserting (30) into (31), we obtain

$$
\left.\left.\left.\left.\| \eta_{\bar{t}}^{n+1}\right]\left.\right|^{2} \leqslant q^{2}(1+C h)^{2}|| \eta_{\bar{t}}^{n}\right]\left.\right|^{2}+\frac{C_{3}(1+C h) h}{(1+0.5 \alpha h)^{2}} \| \Phi_{\bar{t}}^{m, n+1 / 2}\right]\left.\right|^{2}+C h \| \Phi_{\bar{t}}^{m, n+1}\right]\left.\right|^{2} .
$$

Further, noting that $z^{n}=\eta^{n}$ and $z^{n+1}=\eta^{n+1}$, we obtain the required stability inequality.

\subsection{Stability analysis for $E^{m}$}

In this section we will get the recurrence relation for the global errors $E^{m}$.

Let $\Psi_{3}^{m}$ be the truncation error of the discrete amplification condition:

$$
\Psi_{3}^{m}=\left(e^{\alpha z_{a}}-\frac{1}{|q|^{K}}\right) u^{K} .
$$

Lemma 3.2. The finite difference scheme (16) - (17) admits the a priori error estimate

$$
\begin{aligned}
\left.\| E_{\bar{t}}^{m}\right]\left.\right|^{2} \leqslant & \left.\left.\left.\left.e^{3 C z_{a}}\left(\| E_{\bar{t}}^{m-1}\right]\right|^{2}+\left.C z_{a} \max _{1 \leqslant j \leqslant K}\left(|| \Phi_{\bar{t}}^{m, j-1 / 2}\right]\right|^{2}+\| \Phi_{\bar{t}}^{m, j}\right]\left.\right|^{2}\right)\right) \\
& +\left(1+\frac{1}{C z_{a}}\right) \|\left.\Psi_{3 \bar{t}}^{m}\right|^{2} .
\end{aligned}
$$

Proof. Repeatedly using inequality (26), we obtain

$$
\begin{aligned}
\left.\| e_{\bar{t}}^{K}\right]\left.\right|^{2} \leqslant & \left.(1+C h)^{2 K} q^{2 K} \| e_{\bar{t}}^{0}\right]\left.\right|^{2}+C h\left(1+(1+C h)^{2} q^{2}+\cdots+\left((1+C h)^{2} q^{2}\right)^{K-1}\right) \\
& \left.\left.\times\left.\max _{1 \leqslant j \leqslant K}\left(\| \Phi_{\bar{t}}^{m, j-1 / 2}\right]\right|^{2}+\| \Phi_{\bar{t}}^{m, j}\right]\left.\right|^{2}\right) \\
\leqslant & \left.\left.\left.\left.(1+C h)^{2 K} q^{2 K}\left(\| e_{\bar{t}}^{0}\right]\right|^{2}+\left.C z_{a} \max _{1 \leqslant j \leqslant K}\left(\| \Phi_{\bar{t}}^{m, j-1 / 2}\right]\right|^{2}+\| \Phi_{\bar{t}}^{m, j}\right]\left.\right|^{2}\right)\right) .
\end{aligned}
$$

From (32) it follows that

$$
E^{m}=\frac{1}{|q|^{K}} e^{K}+\Psi_{3}^{m}
$$

After simple computations we get

$$
\left.\left.\left.\| E_{\bar{t}}^{m}\right]\left.\right|^{2} \leqslant \frac{1+C z_{a}}{q^{2 K}} \| e_{\bar{t}}^{K}\right]\left.\right|^{2}+\left(1+\frac{1}{C z_{a}}\right) \| \Psi_{3 \bar{t}}^{m}\right]\left.\right|^{2} .
$$

Substituting (34) into inequality (35), we obtain the required estimate. 
Then, Gronwall's lemma and Lemma 3.2 yield the following bound:

$$
\left.\left.\left.\left.\| E_{\bar{t}}^{m}\right]\left.\right|^{2} \leqslant\left.\frac{e^{3 C L}-1}{e^{3 C z_{a}}-1} \max _{1 \leqslant k \leqslant m}\left(\left.C z_{a} e^{3 C z_{a}} \max _{1 \leqslant j \leqslant K}\left(|| \Phi_{\bar{t}}^{k, j-1 / 2}\right]\right|^{2}+\| \Phi_{\bar{t}}^{k, j}\right]\right|^{2}\right)+\left(1+\frac{1}{C z_{a}}\right) \| \Psi_{3 \bar{t}}^{k}\right]\left.\right|^{2}\right) .
$$

Lemma 3.3. For u sufficiently smooth, the truncation errors of finite difference scheme (16) - (17) satisfy the following estimates:

$$
\begin{array}{clrl}
\left.|| \Phi_{\bar{t}}^{m, n-1 / 2}\right] \mid \leqslant C\left(h+\tau^{2}\right), & \left.|| \Phi_{\bar{t}}^{m, n}\right] \mid \leqslant C\left(h+\tau^{2}\right), & 1 \leqslant n \leqslant K, \\
\left.|| \Psi_{3 \bar{t}}^{m}\right] \mid \leqslant C h^{2}, & & 1 \leqslant m \leqslant \frac{M}{K} .
\end{array}
$$

The proof of this lemma is analogous to that of Lemma 2.7.

Then, our main result is the following theorem.

Theorem 3.1. For h sufficiently small, finite difference scheme (16) - (17) has a unique solution, and the global error of the discrete solution satisfies the estimates

$$
\left.\left.\|\left(y^{n}-u^{n}\right)_{\bar{t}}\right] \mid \leqslant C\left(h+\tau^{2}\right), \quad \|\left(V^{m}-u\left(m z_{a}\right)\right)_{\bar{t}}\right] \mid \leqslant C\left(h+\tau^{2}\right) .
$$

The proof of Theorem 3.1 follows from (36) and Lemma 3.3.

\section{Numerical experiments}

In this section, we present the results of numerical experiments where we apply our modified Crank-Nicolson scheme to the problem (1) - (4). The values of the parameters are taken from an example in [12]:

$$
\alpha=17, \quad \mu=1, \quad z_{a}=0.04,
$$

thus, the value of the signal amplification gain is $e^{\alpha z_{a}} \approx 1.97$. The problem is solved in the finite domain $[0, L] \times[0, T]$ with $L=4, T=64$.

The results are presented in Table 1 . The first and second columns list the grid parameters $h$ and $N$, respectively. The third column shows the error, measured in the maximum norm

$$
\|e\|_{C}=\max _{1 \leqslant j<N}\left|u\left(L, t_{j}\right)-y_{j}^{M}\right| .
$$

Table 1 gives rise to the following remarks and conclusions:

- The second-order accuracy is nicely observed if we compare the results on different grids imposing the relation $\tau=C h$ on time-space grid steps.

- We observe that for a fixed values of $\tau$ (or $h$ ), a refinement of the spatial (or time) grid does not generally result in an increased accuracy. A similar effect was noticed in [15], where splitting methods were applied to solve PDE with time-dependent boundary conditions. In our case the explanation of this phenomenon is that different error terms (e.g, temporal error, spatial error, amplification error) may cancel each other. By using the least squares method, we get that

$$
\|e\|_{C} \approx\left|0.036 \tau^{2}-0.025 h^{2}\right|
$$

It is interesting to note that previously such phenomenon was observed for explicit schemes (see, e.g., [7]). 
Table 1. The modified Crank - Nicolson scheme

\begin{tabular}{lcc}
\hline$h$ & $N$ & $\|e\|_{C}$ \\
\hline 0.005 & 512 & 0.00304 \\
0.005 & 1024 & 0.00435 \\
0.005 & 2048 & 0.00583 \\
0.005 & 4096 & 0.00621 \\
0.0025 & 512 & 0.00706 \\
0.0025 & 1024 & 0.00074 \\
0.0025 & 2048 & 0.00109 \\
0.0025 & 4096 & 0.00146 \\
0.00125 & 512 & 0.00820 \\
0.00125 & 1024 & 0.00174 \\
0.00125 & 2048 & 0.00018 \\
0.00125 & 4096 & 0.00028 \\
\hline
\end{tabular}

For the results produced by the splitting difference scheme (16) - (17) we observe a similar behavior.

The authors are grateful to the anonymous referee for several useful comments and helpful remarks.

\section{References}

[1] R. Čiegis, On the convergence in $C$ norm of symmetric difference schemes for nonlinear evolution problems, Lith. Math. J., 32 (1992), No. 2, pp. 187-205.

[2] Raim. Čiegis, Rem. Čiegis, and M. Meilūnas, On a general method for investigation of finite difference schemes, Lith. Math. J., 36 (1996), No. 4, pp. 224-241.

[3] R. Čiegis and V. Pakalnytè, The finite difference scheme for the solution of weakly damped nonlinear Schrödinger equation, Intl. Journal of Applied Science and Computations, 8 (2001), No. 3, pp. 331-340.

[4] R. Čiegis, G. Kairytè, and V. Pakalnytè, On the nonlinear Schrödinger equation with amplification process, Lith. Math. J., 40 (2001), pp. 337-341.

[5] A. Cloot, B. M. Herbst, and J. A. Weideman, A numerical study of the nonlinear Schrödinger equation involving quintic terms, J. Comp. Phys., 44 (1981), pp. 277-288.

[6] M. Delfour, M. Fortin, and G. Payre, Finite difference solutions of a nonlinear Schrödinger equation, J. Comp. Phys., 44 (1981), No. 2, pp. 277-288.

[7] R. E. Ewing and H. Wang, A summary of numerical methods for time-dependent advection-dominated partial differential equations, J. Comput. Appl. Math., 128 (2001), pp. 423-445.

[8] B. M. Herbst and J. A. Weideman, Split step methods for the solution of the nonlinear Schrödinger equation, SIAM J. Numer. Anal., 23 (1986), No. 3, pp. 485-507.

[9] M. Lax, J. H. Battex, and G. P. Agrawal, Channeling of intense electromagnetic beams, J. Appl. Phys., 26 (1981), pp. 109-125.

[10] R. LeVeque and J. Oliger, Numerical methods based on additive splittings for hyperbolic partial differential equations, Math. Comput., 40 (1983), pp. 469-497.

[11] G. Marchuk, The Splitting Method, Nauka, Moscow, 1988, in Russian. 
[12] G. Moebs, A multilevel method for the resolution of a stochastic weakly damped nonlinear Schrödinger equation, Appl. Numer. Math., 26 (1998), No. 3, pp. 353-375.

[13] A. A. Samarskii, Theory of Difference Schemes, Nauka, Moscow, 1989, in Russian; English transl.: The Theory of Difference Schemes, Marcel Dekker, Inc., New York, 2001.

[14] J. M. Sanz-Serna and J. G. Verwer, Conservative and nonconservative schemes for the solution of the nonlinear Schrödinger equation, Report NM-8405, Centre Math. Comp. Sci., Amsterdam, 1984.

[15] B. P. Sommeijer and J. Kok, Splitting methods for three - dimensional bio-chemical transport, Appl. Numer. Math., 21 (1996), pp. 303-320.

[16] G. Strang, On the construction and comparison of difference schemes, SIAM J. Numer. Anal., 5 (1968), pp. 506-517.

[17] T. R. Taha and M. J. Albowitz, Analytical and numerical aspects of certain nonlinear evolution equations. II. Numerical nonlinear Schrödinger type equation, J. Comp. Ph., 55 (1984), pp. 203-230.

[18] J. W. Thomas, Numerical Partial Differential Equations: finite difference methods, Springer, New York-Berlin-Heidelberg-Tokyo, 1995. 